\title{
Impacto da suplementação com ácidos graxos ômega-3 nas subfrações da lipoproteína de alta densidade de indivíduos tabagistas
}

\author{
Impact of omega-3 fatty acid supplementation \\ on high density lipoprotein \\ subfractions of smokers
}

Caroline PAPPIANI ${ }^{1}$

Nágila Raquel Teixeira DAMASCENO1

\section{R E S U M O}

\section{Objetivo}

Avaliar o efeito da suplementação com ômega-3 nas subfrações das lipoproteínas de alta densidade em indivíduos tabagistas.

\section{Métodos}

Ensaio clínico, randomizado, duplo-cego. Foi selecionada uma amostra com 33 tabagistas, de ambos os sexos, com idade entre 30 e 60 anos, suplementados com ômega-3 ( $n=17)$ ou placebo (ácidos graxos ômega-6, $\mathrm{n}=16$ ) por dois meses. As subfrações das lipoproteínas de alta densidade foram analisadas pelo sistema Lipoprint. Os testes estatísticos foram realizados com o auxílio do programa Statistical Package for the Social Sciences, versão 20.0.

\section{Resultados}

A média de idade foi 49 anos, com predominância da raça branca. Após a intervenção, o grupo ômega-3 modificou positivamente o perfil lipídico e as subfrações das lipoproteínas de alta densidade dos tabagistas. Nos modelos de regressão linear testados, o percentual de ácido docosahexaenoico plasmático apresentou associações negativas com o percentual das lipoproteínas de alta densidade-pequena.

\section{Conclusão}

A suplementação com ômega-3 está associada a uma alteração favorável na distribuição das subfrações das lipoproteínas de alta densidade, aumentando as lipoproteínas de alta densidade-grande e diminuindo as lipoproteínas de alta densidade-pequena. Isso reforça a importância do ômega-3 na saúde cardiovascular de indivíduos tabagistas.

Palavras-chave: Ácidos graxos. Hábito de fumar. Lipoproteínas. Ômega-3.

\footnotetext{
1 Universidade de São Paulo, Faculdade de Saúde Pública, Departamento de Nutrição. Av. Dr. Arnaldo, 715, 01246-904, São Paulo, SP, Brasil. Correspondência para/Correspondence to: NRT DAMASCENO.E-mail: <nagila@usp.br>.
} 


\section{A B S T R A C T}

\section{Objective}

To assess the effect of omega-3 supplementation on smokers' high density lipoprotein subfractions.

\section{Methods}

This randomized, double-blind clinical trial included 33 male and female smokers aged 30 to 60 years. The sample took omega-3 fatty acids ( $n=17)$ or placebo (omega- 6 fatty acids, $n=16)$ for two months. The Lipoprint system analyzed the high density lipoprotein subfractions. The statistical tests were performed by the software Statistical Package for the Social Sciences, version 20.0.

\section{Results}

The mean age of the sample was 49 years, and most individuals were white. After the intervention, the lipid profile and high density lipoprotein subfractions of the omega-3 group improved. In the tested linear regression models, the percentage of plasma docosahexaenoic acid was negatively associated with the percentage of small high density lipoprotein.

\section{Conclusion}

Omega-3 supplementation is associated with a favorable change in the distribution of high density lipoprotein subfractions, increasing the large and reducing the small high density lipoproteins. This finding reinforces the importance of omega-3 fatty acids for smokers' cardiovascular health.

Keywords: Fatty acids. Smoking. Lipoproteins. Omega-3.

\section{N T R O D U Ç Ã O}

A doença cardiovascular é a principal causa de morbimortalidade precoce em todo o mundo e, de um modo geral, a aterosclerose é a base fisiopatológica para os eventos clínicos primários e secundários. Esta se desenvolve ao longo de décadas de maneira insidiosa, podendo os primeiros sinais serem fatais ou altamente limitantes ${ }^{1}$.

Além do papel essencial das Low Density Lipoproteins (LDL, Lipoproteínas de Baixa Densidade) na gênese da aterosclerose, é amplamente reconhecido que o baixo conteúdo de colesterol associado às High Density Lipoproteins-cholesterol (HDL-C, Lipoproteínas de Alta Densidade-colesterol) predispõe, de modo independente, à aterosclerose e que o aumento da sua concentração plasmática diminui o risco cardiovascular. No entanto, apesar do papel inquestionável dessas lipoproteínas no desenvolvimento da aterosclerose, a concentração de colesterol nessas partículas não é capaz de explicar todos os eventos cardiovasculares. Nesse sentido, o monitoramento de subfrações lipoproteicas pode fornecer uma estimativa adicional a esse tipo de risco.
Atualmente, o papel antiaterogênico das HDL tem sido amplamente discutido, pois sabe-se que, além do transporte reverso de colesterol, suas propriedades antioxidante, antiplaquetária, antitrombótica, anti-inflamatória e vasodilatadora podem contribuir para sua ação cardioprotetora ${ }^{2}$. Além do conteúdo de colesterol, essas funções estão relacionadas com os diferentes componentes da HDL, os quais influenciam diretamente no tamanho da lipoproteína. Asztalos et al. ${ }^{3}$ mostraram que as partículas grandes de $\mathrm{HDL}\left(\mathrm{HDL}_{\text {GRANDE }}\right)$ estão associadas a um menor risco de doença cardiovascular, enquanto as partículas pequenas $\left(\mathrm{HDL}_{\text {PEQUENA }}\right)$ a um risco aumentado. Os mesmos autores propuseram que o aumento no percentual de $\mathrm{HDL}_{\text {GRANDE }}$ induzido pelo Ômega-3 ( 13 ) estava associado a um elevado potencial anti-inflamatório e que os mecanismos pelos quais o $\omega 3$ aumenta o tamanho da HDL poderiam estar relacionados a alterações na transferência de lipídeos, mediada pela redução da atividade da proteína de transferência de éster de colesterol.

Além dos fatores de risco associados ao perfil lipídico, sabe-se que o sedentarismo, a obesidade e o tabagismo representam fatores de risco modificáveis. Por causa disso, estes têm sido 
o foco da maioria dos programas de prevenção de doença cardiovascular. Embora o tabagismo seja um hábito socialmente tolerável, consequências negativas para a saúde cardiovascular têm sido claramente identificadas, como ações vasculares diretas $^{4}$, estresse oxidativo ${ }^{5}$, efeitos trombogênicos $^{6}$ e dislipidemias secundárias ${ }^{7}$.

O tabagismo é um fator de risco independente para aterosclerose e doença cardiovascular e, embora o seu papel fisiopatológico no risco cardiovascular não esteja totalmente compreendido, a disfunção endotelial mediada pelos radicais livres é considerada um evento chave ${ }^{8}$. Nesse contexto, os lipídeos encontram-se entre os compostos sensíveis a modificações por radicais livres, sendo os ácidos graxos poli-insaturados os principais alvos de processos oxidativos e fonte de produtos radicalares ${ }^{9}$.

Estudos mostram que indivíduos tabagistas têm baixo conteúdo plasmático de $\omega 3$, principalmente Eicosapentaenoic Acid (EPA, Ácido Eicosapentaenoico, 20:5) e Docosahexaenóic Acid (DHA, Ácido Docosahexaenoico, 22:6) ${ }^{10}$. Além disso, baixos níveis de ácidos graxos poli-insaturados foram associados com um maior risco de doença cardiovascular ${ }^{11}$.

Ensaios clínicos demonstram os efeitos benéficos do $\omega 3$ em pacientes com doença cardiovascular, sendo que os mecanismos envolvidos estão relacionados ao seu papel anti-inflamatório ${ }^{12}$, ao efeito na redução plasmática dos triacilglicerois ${ }^{13}$, à redução da frequência cardíaca de repouso ${ }^{14}$, da pressão arterial ${ }^{11}$ e da arritmia $^{15}$ e à melhora da capacidade funcional do miocárdio ${ }^{16}$. Recentemente, Din et al. ${ }^{17}$ demonstraram que o $\omega 3$ melhorou a fibrinólise e a função endotelial em indivíduos tabagistas, considerado um grupo de alto risco para eventos cardiovasculares. Diante disso, o objetivo deste estudo foi avaliar o impacto da suplementação com $\omega 3$ nas subfrações da HDL em indivíduos tabagistas.

\section{MÉ T O D O S}

O ensaio clínico foi do tipo controlado, randomizado, duplo-cego, paralelo, com seguimento de oito semanas e intervenção nutricional baseada na suplementação com ácidos graxos. Para o presente estudo, foi selecionada uma amostra com 33 tabagistas, de ambos os sexos, com idade entre 30 e 60 anos, suplementados com $\omega 3$ ( $n=17)$ ou ômega-6 ( $\omega 6 ; n=16)$. O cálculo amostral foi baseado na diferença entre as médias e desvio-padrão, utilizando $\alpha=0,05$ e poder estatístico de $80 \%$. Desse modo, estimou-se que o tamanho amostral mínimo para observar o efeito do $\omega 3$ sobre o tamanho da HDL seria de 17 indivíduos por grupo. O estudo está cadastrado no Registro Brasileiro de Ensaios Clínicos (REBEC, RBR-2vfhfv).

Os participantes foram recrutados no Hospital Universitário na Universidade de São Paulo. Não foram incluídos gestantes, lactantes, indivíduos que participavam de outros protocolos de investigação, evento cardiovascular prévio (monitorado por meio de eletrocardiograma e avaliação clínica), usuários de drogas ilícitas e alcoolistas. A pesquisa foi aprovada pelos Comitês de Ética em Pesquisa da Faculdade de Saúde Pública da Universidade de São Paulo (n²264) e do Hospital Universitário (n 1126/11) e todos os participantes assinaram o Termo de Consentimento Livre e Esclarecido. O protocolo de intervenção utilizou cápsulas de $\omega 3$ (óleo de peixe composto por $37 \%$ de EPA, $23 \%$ de DHA, $20 \%$ de outros ácidos graxos poli-insaturados e $20 \%$ de ácidos graxos monoinstaurados) e cápsulas de $\omega 6$ (óleo de girassol contendo $70 \%$ de ácido linoleico, 20\% de ácidos graxos monoinsaturados e $10 \%$ de ácidos graxos saturados). As cápsulas foram produzidas, embaladas e aleatorizadas pela empresa Relthy (Indaiatuba, São Paulo), visando garantir que o estudo se mantivesse cego durante toda a intervenção. Os indivíduos foram orientados a ingerir três cápsulas ao dia distribuídas nas três principais refeições (desjejum, almoço e jantar), durante oito semanas. Foi entregue um livro diário para cada participante, no qual foi realizado o registro de efeitos adversos, eventuais mudanças no uso de medicamentos e motivos do não uso da suplementação. O nível de aderência à intervenção foi avaliado por meio da contagem do número de cápsulas restantes ao término da intervenção e confirmado por meio 
da análise de ácidos graxos no plasma, conforme o método de Nimura \& Kinoshita ${ }^{18}$.

Após caracterização demográfica e clínica da amostra, o peso e a altura foram mensurados com precisão de 0,1 kg e 0,1 cm, respectivamente, com equipamentos calibrados e métodos padronizados. A partir desses parâmetros, o Índice de Massa Corporal (IMC) foi calculado $\left(\mathrm{kg} / \mathrm{m}^{2}\right)$. Usando uma fita inelástica, flexível e com precisão de 1,0 mm (TBW ${ }^{\circledR}$, São Paulo), a medida da circunferênciada da cintura foi obtida. A composição corporal foi avaliada por Bioimpedância Elétrica (BIA) (Analyzer ${ }^{\circledR}$ modelo Quantum II, Sistemas RJL, Michigan, Estados Unidos da América) e o percentual de gordura corporal foi estimado por meio do programa Cyprus (RJL Systems ${ }^{\circledR}$, Detroit, Estados Unidos da América) versão 2.5, considerando sexo, idade, peso, altura, nível de atividade física, resistência e reactância.

Após jejum de 12 horas, $20 \mathrm{~mL}$ de sangue foram coletados em tubo seco (soro) contendo Ácido Etilenodiamino Tetra-Acético (EDTA, Ethylenedeamine Tetra Acetic Acid) $(1,0 \mathrm{mg} / \mathrm{mL})$ (plasma) e tubo com fluoreto de sódio (análise de glicose). Adicionou-se ao plasma (3000 rpm, 15 minutos, $4^{\circ} \mathrm{C}$ ) ou soro os seguintes inibidores de proteases: aprotinina $(10 \mathrm{ug} / \mathrm{mL})$, benzamidina (10 mM) e Fluoreto de Fenilmetilsulfonila (PMSF, Phenylmenthylsulfonyl Fluoride) (5 mM) e o antioxidante Hidroxitolueno Butilado (BHT, Butylated Hydroxytoluene) (100 mM). As amostras foram mantidas a $-80^{\circ} \mathrm{C}$ até o momento das análises.

As concentrações plasmáticas de triacilglicerois, colesterol total e HDL-c foram analisadas por meio de kits comerciais (Labtest, Lagoa Santa, Minas Gerais) em sistema semi-automático Cobas $^{\circledR}$. O colesterol associado à LDL foi calculado usando a equação de Friedewald (1972). As apolipoproteínas APO Al e B foram analisadas por método padrão, utilizando o Autokit $\mathrm{APO} \mathrm{Al}^{\circledR} \mathrm{e}$ Autokit APO B ${ }^{\circledR}$ (Wako Chemicals, Inc., Richmond, Virgínia, Estados Unidos). Os kits comerciais da Wako são baseados no método de imunoensaio turbidimétrico para determinação quantitativa de apolipoproteínas específicas no plasma.

As subfrações da HDL foram analisadas pelo sistema Lipoprint (Lipoprint System ${ }^{\circledR}$, Quantimetrix, Redondo Beach, Califórnia, Estados Unidos da América). Foram identificadas 10 subfrações, sendo as partículas da HDL1 a HDL3 classificadas em grandes, HDL4 a HDL7 em intermediárias e HDL8 a HDL10 em pequenas. A glicose plasmática foi analisada por meio de kit comercial (Glicose PAP Liquiform ${ }^{\circledR}$, Labtest, Lagoa Santa, Minas Gerais). Todas as análises foram realizadas em duplicata e os coeficientes de variação intra e inter ensaios ficaram abaixo de 10\%.

Para as variáveis qualitativas, foi utilizado o teste Qui-quadrado e os dados estão apresentados na forma de valor absoluto, seguido da sua respectiva porcentagem. Para a apresentação dos dados quantitativos, assim como para a determinação dos testes utilizados, foi considerado o tipo de distribuição dessas variáveis (teste Shapiro-Wilk). As variáveis com distribuição normal são apresentadas sob a forma de média e desvio-padrão e as demais por mediana $\left(P_{50}\right)$ e intervalos interquartis $\left(\mathrm{P}_{25}\right.$ e $\left.\mathrm{P}_{75}\right)$. A diferença entre os grupos (efeito intervenção) foi testada pela comparação de médias pelo teste $t$ Student para as variáveis paramétricas e Mann Whitney para as variáveis não paramétricas.

Com relação ao efeito tempo (T8 versus T0), em ambos os grupos foram avaliados pelos testes $t$ Student pareado e Wilcoxon, a depender da normalidade da variável. Foi utilizada a técnica de análise de ensaios aleatorizados e controlados "intenção-de-tratar", em que os indivíduos são comparados - em termos de resultados finais -, dentro dos grupos para os quais foram inicialmente aleatorizados, independentemente de terem desistido do estudo (drop-outs). Para avaliar a influência do $\omega 3$ nas subfrações das HDL (variáveis dependentes), foram realizadas análises de regressão linear simples e múltipla. Como variável independente, foi testado o percentual de $E P A$, de DHA e a soma (EPA + DHA) incorporados ao plasma após intervenção. No entanto, a va- 
riável que atendeu aos pressupostos da regressão linear foi o percentual de DHA. Essa variável independente foi testada na forma bruta e ajustada pela idade (modelo 1), idade e sexo (modelo 2) e idade, sexo e hipertensão arterial sistêmica autorrelatada (modelo 3). Os testes estatísticos foram realizados com o auxílio do programa Statistical Package for the Social Sciences (SPSS Inc., Chicago, Illinois, Estados Unidos da América), versão 20.0. O valor de significância considerado foi de $p<0,05$.

\section{RES U L T A D O S}

Na Tabela 1 estão descritas as características clínicas, demográficas e antropométricas dos tabagistas. Em ambos os grupos, a média da idade foi de 49 anos, com predominância da raça branca ( $\omega 3=76 \%$ e $\omega 6=63 \%$ ). Observou-se que os grupos também foram semelhantes em relação ao sexo ( $\omega 3=53 \%$ masculino e $\omega 6=50 \%$ ). Quanto às demais variáveis, os grupos também foram considerados estatisticamente semelhantes, sendo que os parâmetros antropométricos eviden- ciaram presença de excesso de peso, bem como elevada circunferência da cintura e percentual de gordura corporal. Os resultados do percentual de adesão dos participantes à intervenção mostraram que $84,0 \%$ das cápsulas de $\omega 3$ foram ingeridas, sendo esse percentual associado ao aumento de $87,5 \%$ do DHA e $70,0 \%$ do EPA no plasma desses indivíduos (dados não apresentados).

O perfil bioquímico está apresentado na Tabela 2. Comparando o efeito intervenção, no momento basal os grupos foram considerados semelhantes para todas as variáveis. No entanto, após oito semanas, houve diferença no colesterol total, LDL-c, não HDL-c e razão LDL-c por apolipoproteína B (LDL-C/APOB), colesterol total/HDL-c e $\mathrm{LDL}-\mathrm{C} / \mathrm{HDL}-\mathrm{C}$, indicando que o grupo $\omega 3$ modificou positivamente o perfil lipídico dos tabagistas em comparação ao grupo $\omega 6$. Além disso, avaliando o efeito tempo, observou-se que no grupo $\omega 3$ houve redução do colesterol total, não-HDL-C, triacilglicerois, LDL-C/APOB, triacilglicerois/HDL-C, colesterol total/HDL-c e LDL-C/HDL-C, enquanto o grupo $\omega 6$ não apresentou mudanças significativas.

Tabela 1. Características clínicas, demográficas e antropométricas de indivíduos tabagistas. São Paulo (SP), 2015.

\begin{tabular}{|c|c|c|c|c|c|}
\hline \multirow{2}{*}{$\frac{\text { Variáveis }^{*}}{\text { Idade (anos) }}$} & \multicolumn{2}{|c|}{ Ômega-3 ( $n=17)$} & \multicolumn{2}{|c|}{ Ômega-6 ( $n=16)$} & \multirow{2}{*}{$\frac{p}{0,976}$} \\
\hline & 49,3 & 9,5 & 49,2 & 10,3 & \\
\hline \multicolumn{6}{|l|}{ Sexo (\%) } \\
\hline Masculino & 9,0 & 53,0 & 8,0 & 50,0 & 0,866 \\
\hline Feminino & 8,0 & 47,0 & 8,0 & 50,0 & \\
\hline \multicolumn{6}{|l|}{ Raça (\%) } \\
\hline Branca & 13,0 & 76,0 & 10,0 & 63,0 & 0,199 \\
\hline Negra & 2,0 & 12,0 & - & - & \\
\hline Outras & 2,0 & 12,0 & 6,0 & 36,0 & \\
\hline$I M C\left(\mathrm{~kg} / \mathrm{m}^{2}\right)$ & 28,7 & 5,9 & 29,4 & 5,8 & 0,739 \\
\hline $\mathrm{CC}(\mathrm{cm})$ & 97,7 & 16,1 & 97,6 & 9,8 & 0,988 \\
\hline GC (\%) & 30,9 & 12,7 & 32,3 & 13,2 & 0,770 \\
\hline PAS $(\mathrm{mmHg})$ & 127,8 & 19,1 & 131,4 & 20,4 & 0,604 \\
\hline $\mathrm{PAD}(\mathrm{mmHg})$ & 78,4 & 10,2 & 80,8 & 9,2 & 0,484 \\
\hline
\end{tabular}

Nota: *Resultados apresentados em média e desvio-padrão para as variáveis quantitativas e em valor absoluto (n) e frequência (\%) para as variáveis qualitativas. Diferença entre os grupos testada pelo teste $t$ Student para as variáveis quantitativas e teste Qui-quadrado para as variáveis qualitativas. Valor de significância $p<0,05$.

IMC: Índice de Massa Corporal; CC: Circunferência da Cintura; GC: Percentual de Gordura Corporal; PAS: Pressão Arterial Sistólica; PAD: Pressão Arterial Diastólica; M: Média; DP: Desvio-Padrão. 
Tabela 2. Características bioquímicas de indivíduos tabagistas. São Paulo (SP), 2015.

\begin{tabular}{|c|c|c|c|c|c|c|c|c|c|c|}
\hline \multirow{3}{*}{$\begin{array}{l}\text { Variáveis (mg/dL) } \\
\text { Colesterol total }\end{array}$} & \multicolumn{4}{|c|}{ Ômega-3 (n=17) } & \multicolumn{4}{|c|}{ Ômega-6 (n=16) } & \multirow{3}{*}{$\frac{p^{*}}{0,298}$} & \multirow{3}{*}{$\begin{array}{c}p^{* *} \\
0,02\end{array}$} \\
\hline & \multicolumn{2}{|c|}{ TO } & \multicolumn{2}{|c|}{ T8 } & \multicolumn{2}{|c|}{ TO } & \multicolumn{2}{|c|}{ T8 } & & \\
\hline & 195,7 & 32 & 173,29 & 28 & 212,20 & 55 & 211,8 & 55 & & \\
\hline LDL-C & 126,82 & 32 & 109,59 & $25^{a}$ & 139,10 & 48 & 144,3 & 49 & 0,396 & 0,039 \\
\hline $\mathrm{HDL}-\mathrm{C}$ & 37,29 & 10 & 39,12 & 10 & 34,10 & 7 & 35,1 & 7 & 0,297 & 0,21 \\
\hline $\mathrm{nHDL}-\mathrm{c}$ & 158,88 & 31 & 134,06 & $24^{a}$ & 178,60 & 55 & 182,1 & 50 & 0,221 & 0,002 \\
\hline APOAI & 134,69 & 25 & 136,61 & 29 & 131,10 & 30 & 133,5 & 25 & 0,715 & 0,747 \\
\hline APOB & 99,90 & 23 & 104,47 & 19 & 109,40 & 26 & 114,6 & 24 & 0,276 & 0,187 \\
\hline HDL-C/APOAI & 0,27 & 0,05 & 0,28 & 0,05 & 0,26 & 0,06 & 0,26 & 0,04 & 0,673 & 0,192 \\
\hline LDL-C/APOB & 1,30 & 0,39 & 1,05 & $0,16^{\mathrm{a}}$ & 1,26 & 0,2 & 1,21 & 0,2 & 0,732 & 0,019 \\
\hline APOAI/APOB & 0,75 & 0,17 & 0,78 & 0,14 & 0,87 & 0,29 & 0,87 & 0,17 & 0,156 & 0,107 \\
\hline TAG & 143,00 & $107-181$ & 122,00 & 63-186 & 158,00 & $120-211$ & 112 & $84-129$ & 0,517 & 0,954 \\
\hline TAG/HDL-C & 4,00 & $3-7$ & 2,00 & $1-6$ & 5,00 & $3-7$ & 5 & $3-7$ & 0,231 & 0,122 \\
\hline CT/HDL-C & 5,00 & $4-7$ & 5,00 & $4-5^{a}$ & 6,00 & $5-8$ & 6 & $5-7$ & 0,176 & 0,001 \\
\hline LDL-C/HDL-C & 3,00 & $3-5$ & 3,00 & $2-3^{a}$ & 4,00 & $3-5$ & 4 & $3-5$ & 0,279 & 0,008 \\
\hline Glicemia & 93,00 & 88-97 & 95,00 & 89-104 & 95,00 & $90-102$ & 93 & $87-102$ & 0,528 & 0,564 \\
\hline
\end{tabular}

Nota: *Diferença testada no momento basal (T0) (ômega-3 versus ômega-6); **Diferença testada após a intervenção (T8) (ômega-3 versus ômega6); "Diferença testada antes e após a intervenção (T8 versus T0). Valores em negrito mostram diferenças significativas entre os grupos. Resultados apresentados em média (desvio padrão) para as variáveis paramétricas e em mediana (intervalos interquartis) para as variáveis não paramétricas. Diferença intragrupo (efeito tempo) testada pelo teste $t$ Student pareado para as variáveis paramétricas e teste Wilcoxon para as variáveis não paramétricas. Valor de significância $p<0,05$. Diferença entre os grupos testada pelo teste $t$ Student para as variáveis paramétricas e teste Mann Whitney para as não paramétricas.

CT: Colesterol Total; LDL-c: Low Density Lipoprotein-cholesterol; HDL-c: High Density Lipoprotein-cholesterol; HDL-c: Fração de colesterol não HDL-c; APOAl: Apolipoproteína Al; APOB: Apolipoproteína B; HDL-C/APOAl: Razão entre HDL-c e APOAl; LDL-C/APOB: Razão entre LDL-c e APOB; APOAl/APOB: Razão entre apolipoproteínas; TAG: Triacilglicerois; TAG/HDL-c: Razão entre Triacilglicerois e HDL-C; CT/HDL-c: Razão Entre Colesterol Total e HDL-c; LDL-C/HDL-c: Razão entre LDL-c e HDL-c; M: Média; DP: Desvio-Padrão.

O percentual e a concentração das subfrações das HDL estão apresentados na Tabela 3. No momento basal, os grupos foram considerados semelhantes para todas as variáveis, com exceção do percentual da subfração HDL10 e HDL PEQUeNA $_{\text {. }}$ Contudo, ao avaliarmos o efeito intervenção, observou-se que o grupo $\omega 3$ promoveu aumento da $\mathrm{HDL}_{\text {GRANDE' }}$ sendo essa variação associada à elevação da subfração HDL de maior tamanho (HDL1). Perfil inverso foi encontrado na $\mathrm{HDL}_{\text {PEQUENA' }}$ cuja subfração HDL10 foi a que apresentou maior redução. O efeito positivo do $\omega 3$ se manteve após o ajuste do percentual das subfrações pelo conteúdo de colesterol associado à HDL. O efeito do $\omega 3$ no tamanho das partículas foi confirmado nas comparações entre os grupos, visto que houve ausência do efeito tempo ( $p>0,05$ para ambas intervenções). A Figura 1 mostra as principais mudanças nas subfrações das HDL induzidas pelo $\omega 3$ após oito semanas de intervenção.

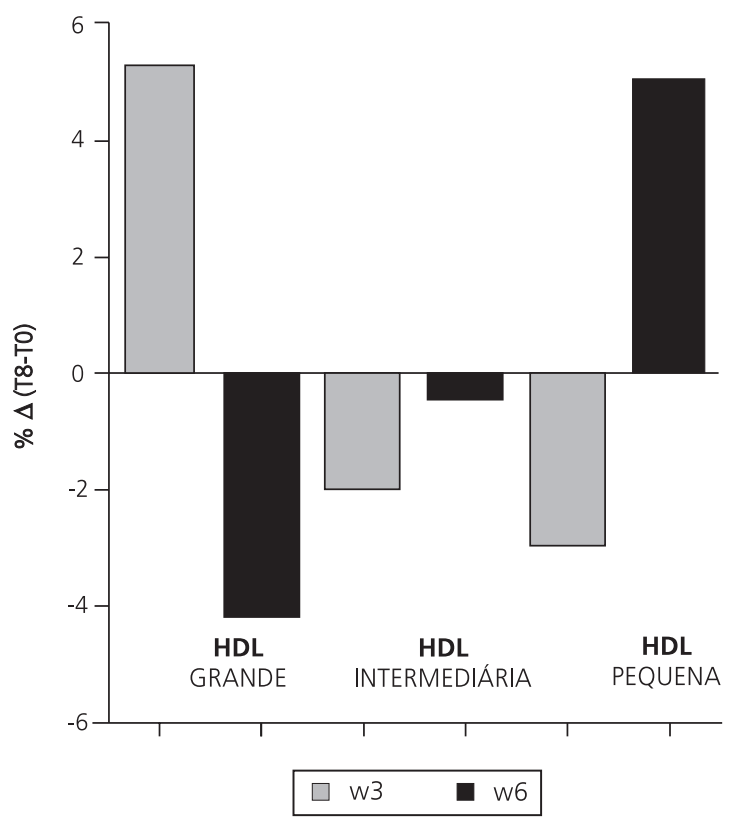

Figura 1. Percentual de variação nas subfrações das High Density Lipoproteins após oito semanas de intervenção, segun-

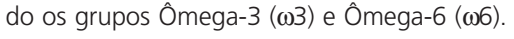


Tabela 3. Subfrações das High Density Lipoproteins (HDL) de indivíduos tabagistas. São Paulo (SP), 2015.

\begin{tabular}{|c|c|c|c|c|c|c|c|c|c|c|}
\hline \multirow{2}{*}{ Variáveis } & \multicolumn{4}{|c|}{ Ômega-3 ( $n=17)$} & \multicolumn{4}{|c|}{ Ômega-6 (n=16) } & \multirow{2}{*}{$p^{*}$} & \multirow{2}{*}{$p^{* *}$} \\
\hline & \multicolumn{2}{|c|}{ TO } & \multicolumn{2}{|c|}{ T8 } & \multicolumn{2}{|c|}{ TO } & \multicolumn{2}{|c|}{ T8 } & & \\
\hline \multicolumn{11}{|l|}{ Percentual (\%) } \\
\hline HDL1 & 9,5 & 3,8 & 10,2 & 2,9 & 8,5 & 3,7 & 7,6 & 3,2 & 0,466 & 0,018 \\
\hline HDL2 & 12,2 & 4,2 & 12,5 & 5,3 & 9,7 & 2,9 & 9,7 & 2,9 & 0,065 & 0,070 \\
\hline HDL3 & 6,8 & 2,2 & 7,2 & 2,5 & 5,9 & 1,7 & 5,9 & 1,7 & 0,250 & 0,106 \\
\hline HDL4 & 9,6 & 1,8 & 9,4 & 1,4 & 8,7 & 1,4 & 8,5 & 1,2 & 0,118 & 0,004 \\
\hline HDL5 & 11,5 & 1,7 & 11,2 & 1,6 & 11,1 & 1,0 & 10,8 & 1,2 & 0,496 & 0,460 \\
\hline HDL6 & 22,4 & 2,9 & 21,9 & 3,3 & 21,9 & 2,0 & 21,9 & 2,0 & 0,572 & 0,999 \\
\hline HDL7 & 7,8 & 1,5 & 7,7 & 1,5 & 7,9 & 1,1 & 8,2 & 0,8 & 0,664 & 0,242 \\
\hline HDL8 & 7,6 & 1,7 & 7,6 & 2,0 & 8,2 & 1,4 & 8,6 & 1,2 & 0,258 & 0,101 \\
\hline HDL9 & 6,6 & 1,9 & 6,2 & 1,8 & 6,4 & 1,3 & 6,7 & 1,4 & 0,785 & 0,410 \\
\hline HDL10 & 6,0 & 3,6 & 5,9 & 3,4 & 11,3 & 4,5 & 11,9 & 4,5 & 0,001 & $<0,001$ \\
\hline $\mathrm{HDL}_{\text {GRANDE }}$ & 28,4 & 8,3 & 29,9 & 9,8 & 24,2 & 7,2 & 23,2 & 6,6 & 0,132 & 0,029 \\
\hline $\mathrm{HDL}_{\text {INTERMEDIARIA }}$ & 51,3 & 4,2 & 50,3 & 5,5 & 49,7 & 2,3 & 49,5 & 2,3 & 0,204 & 0,590 \\
\hline$H D L_{\text {PEQUENA }}$ & 20,3 & 6,7 & 19,7 & 6,5 & 25,9 & 6,1 & 27,2 & 5,9 & 0,017 & 0,002 \\
\hline \multicolumn{11}{|c|}{ Concentração (mg/dL) } \\
\hline HDL1 & 3,0 & $2-5$ & 4,0 & $3-6$ & 3,0 & $2-4$ & 3,0 & $2-3$ & 0,292 & 0,010 \\
\hline HDL2 & 4,0 & $2-7$ & 4,0 & $2-7$ & 3,0 & $2-4$ & 3,0 & $2-5$ & 0,090 & 0,102 \\
\hline HDL3 & 2,0 & $1-3$ & 2,0 & $2-4$ & 2,0 & $1-3$ & 2,0 & $1-3$ & 0,496 & 0,143 \\
\hline HDL4 & 4,0 & $2-4$ & 4,0 & $3-5$ & 3,0 & $2-3$ & 3,0 & $2-3$ & 0,118 & 0,041 \\
\hline HDL5 & 4,0 & $3-5$ & 4,0 & $3-5$ & 4,0 & $3-4$ & 4,0 & $3-4$ & 0,417 & 0,198 \\
\hline HDL6 & 8,0 & $7-10$ & 8,0 & $7-10$ & 7,0 & $6-9$ & 8,0 & $7-9$ & 0,226 & 0,473 \\
\hline HDL7 & 3,0 & $2-3$ & 3,0 & $2-4$ & 3,0 & $2-3$ & 3,0 & $3-3$ & 0,642 & 0,953 \\
\hline HDL8 & 3,0 & $2-3$ & 3,0 & $2-4$ & 3,0 & $2-3$ & 3,0 & $3-4$ & 0,691 & 0,521 \\
\hline HDL9 & 2,0 & $2-3$ & 2,0 & $2-3$ & 2,0 & $2-2$ & 2,0 & $2-3$ & 0,300 & 0,969 \\
\hline HDL10 & 2,0 & $1-4$ & 2,0 & $2-3$ & 4,0 & $3-5$ & 4,0 & $3-6$ & 0,004 & 0,006 \\
\hline $\mathrm{HDL}_{\text {GRANDE }}$ & 10,0 & $6-14$ & 10,0 & $7-15$ & 7,0 & $5-10$ & 7,0 & $5-10$ & 0,138 & 0,045 \\
\hline $\mathrm{HDL}_{\text {INTERMEDIARIA }}$ & 18,0 & $16-22$ & 19,0 & $15-23$ & 15,0 & $15-19$ & 16,0 & $15-20$ & 0,102 & 0,286 \\
\hline $\mathrm{HDL}_{\text {PEQUENA }}$ & 7,0 & $5-10$ & 7,0 & $6-9$ & 9,0 & $7-10$ & 10,0 & $7-11$ & 0,099 & 0,013 \\
\hline
\end{tabular}

Nota: "Diferença testada no momento basal (T0) (ômega-3 versus ômega-6); **Diferença testada após a intervenção (T8) (ômega-3 versus ômega-6). Valores em negrito mostram diferenças significativas entre os tempos ou grupos. Resultados apresentados em média (desvio-padrão) para as variáveis paramétricas e em mediana (intervalo interquartil) para as variáveis não paramétricas. $\mathrm{HDL}_{\text {GRANDE: }}$ partículas grandes de $\mathrm{HDL}$ (1 a 3);

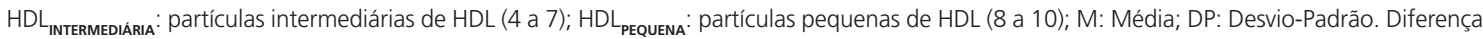
entre os grupos testada pelo teste $t$ Student para as variáveis paramétricas e teste Mann Whitney para as variáveis não paramétricas, com valor de significância $p<0,05$.

Foram testados todos os pressupostos para inclusão de variáveis nos modelos de regressão linear, sendo que o percentual das HDL1, HDL2, HDL3, HDL GRANDE, $_{\text {HDL8, HDL9, HDL10 e HDL }}$ PEQUeNA preencheram os requisitos para a modelagem. No modelo de regressão linear bruto, observou-se que o percentual de DHA plasmático influenciou de maneira significativa as variáveis dependentes: $\mathrm{HDL}_{\text {GRANDE' }^{\prime}} \mathrm{HDL} 8, \mathrm{HDL}$, HDL10 e HDL PEQUENA . Após a intervenção, o DHA plasmático foi associado à redução de $18 \%$ da variação no percentual das
HDL8, $19 \%$ das HDL9, 32\% das HDL10, 32\% das $\mathrm{HDL}_{\text {PEQUENA }}$ e aumento de $17 \%$ da $\mathrm{HDL}_{\text {GRANDE }}$ dos tabagistas. Em todos os modelos testados, independentemente do ajuste, o percentual de DHA plasmático apresentou associações negativas com o percentual das HDL10 e HDL PEQUENA' ou seja, nos modelos múltiplos, as variações permaneceram significativas, independentemente da inclusão das variáveis de ajuste (idade, sexo e hipertensão arterial sistêmica), conforme apresentado na Tabela 4. 
Tabela 4. Modelos de regressão linear para avaliação da influência do ômega-3 nas subfrações das High Density Lipoproteins (HDL) de indivíduos tabagistas. São Paulo (SP), 2015.

\begin{tabular}{|c|c|c|c|c|}
\hline Modelos & $\beta$ & IC95\% & $\mathrm{R}^{2}$ & $p$ \\
\hline \multicolumn{5}{|l|}{ Bruto } \\
\hline$\% \mathrm{HDL}_{\text {GRANDE }}$ & 0,413 & 0,$02 ; 1,76$ & 0,171 & 0,045 \\
\hline$\%$ HDL8 & $-0,424$ & $-0,37 ;-0,01$ & 0,179 & 0,039 \\
\hline$\%$ HDL9 & $-0,440$ & $-0,34 ;-0,02$ & 0,193 & 0,032 \\
\hline \%HDL10 & $-0,566$ & $-0,91 ;-0,19$ & 0,320 & 0,004 \\
\hline$\% H D L_{\text {PEQUENA }}$ & $-0,564$ & $-1,52 ;-0,32$ & 0,318 & 0,004 \\
\hline \multicolumn{5}{|l|}{ Modelo 1} \\
\hline$\% \mathrm{HDL}_{\text {GRANDE }}$ & 0,438 & 0,$02 ; 1,86$ & 0,178 & 0,127 \\
\hline \%HDL8 & $-0,382$ & $-0,36 ; 0,02$ & 0,201 & 0,095 \\
\hline \%HDL9 & $-0,420$ & $-0,34 ; 0,01$ & 0,198 & 0,099 \\
\hline \%HDL10 & $-0,560$ & $-0,93 ;-0,16$ & 0,321 & 0,017 \\
\hline$\% \mathrm{HDL}_{\text {PEQUENA }}$ & $-0,544$ & $-1,53 ;-0,25$ & 0,323 & 0,017 \\
\hline \multicolumn{5}{|l|}{ Modelo 2} \\
\hline \%HDL10 & $-0,595$ & $-0,98 ;-0,18$ & 0,336 & 0,039 \\
\hline$\% \mathrm{HDL}_{\text {PEQUENA }}$ & $-0,536$ & $-1,56 ;-0,20$ & 0,324 & 0,046 \\
\hline \multicolumn{5}{|l|}{ Modelo 3} \\
\hline \%HDL10 & $-0,633$ & $-1,02 ;-0,22$ & 0,393 & 0,041 \\
\hline$\% H D L_{\text {PEQUENA }}$ & $-0,591$ & $-1,61 ;-0,32$ & 0,440 & 0,021 \\
\hline
\end{tabular}

Nota: Modelo 1: Ajustado pela idade; Modelo 2: Ajustado pela idade e sexo. Modelo 3: Ajustado pela idade, sexo e hipertensão arterial sistêmica. Valores em negrito mostram diferenças significativa.

\section{I S C U S S Ã O}

Aproximadamente $30 \%$ de todas as mortes anuais por doenças cardiovasculares nos Estados Unidos da América são atribuíveis ao tabagismo ${ }^{19}$. Em adição, dados epidemiológicos obtidos com populações da América do Sul, América Central e América do Norte mostram que as mortes por doença cardiovascular atribuídas ao tabagismo são duas vezes mais prevalentes do que aquelas atribuídas ao câncer de pulmão e doenças respiratórias ${ }^{20}$. Esse elevado impacto negativo do tabagismo sobre a saúde cardiovascular é resultante do efeito nocivo direto do fumo, mas também da ação sinérgica com outros fatores de risco, aumentando a incidência de doença cardiovascular e outras doenças crônicas.

Os resultados apresentados no presente estudo mostraram que o $\omega 3$ contribuiu para a redução do risco cardiometabólico de indivíduos tabagistas pela modificação de vários parâmetros lipídicos. De acordo com Kajikawa et al. ${ }^{7}$, o tabagismo está associado ao aumento nas concen- trações plasmáticas de colesterol total e LDL-C, em detrimento da redução no HDL-c. O presente estudo mostrou que a suplementação com $\omega 3$ foi capaz de melhorar o perfil lipídico de tabagistas uma vez que, após oito semanas de intervenção, houve redução plasmática de colesterol total e LDL-c. Resultado semelhante foi relatado pelo estudo de Ebrahimi et al. ${ }^{21}$, no qual, após seis meses de suplementação com EPA (180 mg) e DHA (120 mg) em pacientes com síndrome metabólica, foi encontrada redução plasmática de colesterol total e LDL-c, mas não foram observadas modificações no HDL-C.

Em uma meta-análise com ensaios clínicos randomizados, placebo-controle, Jacobson et al. ${ }^{22}$ mostraram que o EPA poderia reduzir, mas que o DHA seria capaz de aumentar o LDL-c. No presente estudo, a suplementação de $\omega 3$ foi realizada de forma concomitante, de forma a fornecer 1,11 $\mathrm{g}$ de EPA e 0,69 $\mathrm{g}$ de DHA por dia. Sendo assim, não foi possível avaliar os potenciais efeitos diferenciais entre os dois ácidos graxos. 
Apesar do impacto positivo do $\omega 3$ no perfil lipídico clássico, sabe-se que as variações nesses parâmetros não permitem avaliar as modificações qualitativas das lipoproteínas. Asztalos et al. ${ }^{3}$ mostraram que partículas grandes de HDL estão associadas a um menor risco de doença cardiovascular, enquanto que as partículas pequenas a um maior risco. Os resultados obtidos nessas pesquisas são reforçados pela observação de que a funcionalidade da HDL é fortemente influenciada pela distribuição de suas subfrações e que o transporte reverso do colesterol, considerado a principal função biológica da HDL, se associa às partículas grandes 3,23 .

De acordo com o estudo de de Roos et al. ${ }^{24}$ o uso de óleo de peixe $(1,5 \mathrm{~g} / \mathrm{dia})$ em indivíduos saudáveis promoveu aumento de partículas grandes de HDL. Já os resultados descritos por Neff et al. ${ }^{25} \mathrm{em}$ indivíduos com excesso de peso e por Kelley et al. ${ }^{26}$ em indivíduos com hipertrigliceridemia mostraram que a suplementação com DHA (2-3 g/d) contribuiu para o aumento dessas mesmas subfrações.

Os resultados desta pesquisa confirmam o papel protetor do $\omega 3$ no aumento do percentual de partículas de HDL grandes e na simultânea redução de partículas pequenas de HDL em indivíduos tabagistas. Erkkilä et al. ${ }^{27}$ também encontraram efeitos positivos no tamanho da partícula de HDL em um estudo com indivíduos com doença coronária após oito semanas de intervenção baseada na ingestão de peixes fonte de $\omega 3$, pelo menos quatro vezes por semana.

Atualmente, sabe-se que a composição da $\mathrm{HDL}$, que vai além do conteúdo de colesterol, e o tamanho dessa partícula determinam sua funcionalidade. Dessa forma as funções da HDL incluem o transporte reverso do colesterol, função antioxidante, antiplaquetária, antitrombótica, anti-inflamatória e vasodilatadora² ${ }^{2}$ Embora o presente estudo não tenha avaliado aspectos funcionais da HDL, o aumento no percentual de HDL grande induzido pelo $\omega 3$ tem sido associado ao maior potencial anti-inflamatório ${ }^{3}$.
O tabagismo e os produtos da combustão do tabaco (nicotina, monóxido de carbono e outros produtos gasosos e radicalares) têm potente ação inflamatória. Nesse contexto, o $\omega 3$, ao modular vias moleculares anti-inflamatórias (ciclooxigenases e lipooxigenases), mediadores celulares (receptor Toll-like e NF-kB) e modificar o metabolismo lipídico, apresenta múltiplas ações de proteção à saúde cardiovascular do indivíduo tabagista. Particularmente em relação ao papel anti-inflamatório da HDL, tem sido descrito que indivíduos tabagistas têm menos HDL e APO Al, ou seja, parâmetros particularmente reduzidos nas HDL pequenas ${ }^{28}$. Na presente pesquisa, não foram observadas alterações na concentração total de HDL-c e APO Al e suas interrrelações. Entretanto, o $\omega 3$ foi capaz de reduzir as partículas de HDL pequenas, com destaque para as subfrações HDL10.

Ao longo das últimas duas décadas, inúmeros estudos identificaram efeitos positivos das HDL sobre as funções das células endoteliais. Em paralelo, sabe-se que indivíduos tabagistas apresentam elevada instabilidade vascular associada ao aumento de moléculas de adesão (moléculas de adesão à intercélular - ICAM, molécula de adesão à células vasculares - VCAM e e-Selectina). No endotélio, as HDL inibem marcadores inflamatórios (interleucina 6 - IL-6 e fator denecrose tumoral alfa - TNF- $\alpha$ ) e moléculas de adesão ${ }^{28}$. Portanto, os resultados obtidos no presente estudo têm potencial de contribuir para uma melhor função endotelial em indivíduos tabagistas por meio dos efeitos pleiotrópicos do $\omega 3$ em nível inflamatório, qualidade das subfrações da HDL e ação antiplaquetária. Definir especificamente o papel funcional das subfrações de HDL ainda é uma lacuna na literatura, sobretudo no que diz respeito aos ensaios clínicos.

Desse modo, indivíduos tabagistas podem se beneficiar da suplementação com $\omega 3$ e do consumo de alimentos fontes desse ácido graxo devido aos diversos mecanismos descritos e que são potencialmente modulados positivamente pelas partículas de HDL grandes, conforme demons- 
traram os resultados desta pesquisa. Destaca-se, também, o fato dos participantes inclusos no estudo fazerem uso de estatinas durante o período de intervenção. Esses indivíduos não foram excluídos e o parâmetro não foi considerado um viés, visto que a modulação do HDL-c com estatina é limitada, além do fato que a relação entre HDL e risco para doenças cardiovasculares permanece inalterada ${ }^{29}$.

Essas observações reforçam a importância do $\omega 3$ na modulação não farmacológica da HDL. Isso porque, considerando que não houve modificação no esquema terapêutico durante o período de intervenção, os resultados obtidos confirmam a importância da suplementação com $\omega 3$ como parte da estratégia global de tratamento de indivíduos tabagistas com dislipidemias.

No presente estudo, o tamanho da amostra poderia ser considerado uma limitação. No entanto, o número de indivíduos é comparável com estudos anteriores ${ }^{30}$ e foi suficientemente adequado para confirmar estatisticamente os benefícios do $\omega 3$ em pessoas tabagista. A duração da pesquisa foi de oito semanas, representando um período suficiente para promover aumento de ácidos graxos circulantes e modular positivamente o metabolismo lipídico.

\section{O N CLUS Ã O}

Os resultados aqui apresentados mostram que a suplementação com $\omega 3$ modificou positivamente o perfil lipídico (colesterol total e LDL-c), bem como melhorou a distribuição das subfrações de HDL em tabagistas, sendo que as maiores partículas (HDL1) aumentaram e as menores (HDL10) foram reduzidas. Esse resultado contribui para reforçar a importância do $\omega 3$ na saúde cardiovascular de indivíduos tabagistas, mesmo sob tratamento com estatina.

\section{A GRADECIMENTOS}

Ao Instituto Nacional de Ciências e Tecnologias de Fluídos Complexos (2010-2014), Núcleo de Apoio à Pesquisa em Fluídos Complexos Universidade de São Paulo (2011-2016) e à Fundação de Amparo à Pesquisa do Estado de São Paulo, Processo 2013/14863-0.

\section{COLABORADORES}

C PAPPIANI concepção e desenho da pesquisa, obtenção de dados, análise e interpretação dos dados, análise estatística, redação do manuscrito, revisão crítica do manuscrito quanto ao conteúdo intelectual importante. NRT DAMASCENO concepção e desenho da pesquisa, obtenção de dados, análise e interpretação dos dados, obtenção de financiamento, redação do manuscrito, revisão crítica do manuscrito quanto ao conteúdo intelectual importante.

\section{REFERÊ N CIAS}

1. World Health Organization. Prevention of cardiovascular disease: Guidelines for assessment and management of cardiovascular risk. Geneva: WHO; 2007.

2. Gordon SM, Davidson WS. Apolipoprotein A-I mimetics and high-density lipoprotein function. Curr Opin Endocrinol Diabetes Obes. 2012; 19(2):109-14. http://dx.doi.org/10.1097/MED.0b $013 \mathrm{e} 32835056 \mathrm{~d} 4$

3. Asztalos BF, de la Llera-Moya M, Dallal GE, Horvath $\mathrm{KV}$, Schaefer EJ, Rothblat GH. Differential effects of HDL subpopulations on cellular ABCA1- and SRBI-mediated cholesterol efflux. J Lipid Res. 2005; 46(10):2246-53. http://dx.doi.org/10.1194/jlr. M500187-JLR200

4. Erhardt L. Cigarette smoking: An undertreated risk factor for cardiovascular disease. Atherosclerosis. 2009; 205(1):23-32. http://dx.doi.org/ 10.1016/j. atherosclerosis.2009.01.007

5. Freikman I, Amer J, Cohen JS, Ringel I, Fibach E. Oxidative stress causes membrane phospholipid rearrangement and shedding from $\mathrm{RBC}$ membranes: An NMR study. Biochim Biophys Acta. 2008; 1778(10):2388-94. http://dx.doi.org/10.1016/j. bbamem.2008.06.008

6. Fisher SD, Zareba W, Moss AJ, Marder VJ, Sparks $\mathrm{CE}$, Hochman J, et al. Effect of smoking on lipid and thrombogenic factors two months after acute myocardial infarction. Am J Cardiol. 2000; 86(8):813-8. http://dx.doi.org/10.1016/S0002-9 149(00)01098-5

7. Kajikawa S, Harada T, Kawashima A, Imada K, Mizuguchi K. Highly purified eicosapentaenoic acid 
prevents the progression of hepatic steatosis by repressing monounsaturated fatty acid synthesis in high-fat/high-sucrose diet-fed mice. Prostaglandins Leukot Essent Fatty Acids. 2009; 80(4):229-38. http://dx.doi.org/10.1016/j.bbalip.2009.01.014

8. Rahman MM, Laher I. Structural and functional alteration of blood vessels caused by cigarette smoking: An overview of molecular mechanisms. Curr Vasc Pharmacol. 2007; 5(4):276-92. http://dx. doi.org/10.2174/157016107782023406

9. Reddy RD, Yao JK. Environmental factors and membrane polyunsaturated fatty acids in schizophrenia. Prostaglandins Leukot Essent Fatty Acids. 2003; 69(6):385-91. http://dx.doi.org/10. 1016/j.plefa.2003.08.009

10. lonescu N, de Freitas C, Bueno AA. Perturbations in blood phosphatidylcholine and sphingomyelin fatty acid composition in a sample population of cigarette smokers. Indian J Clin Biochem. 2013; 28(4):361-7. http://dx.doi.org/10.1007/s12291-0 13-0327-y

11. Mozaffarian D. Does alpha-linolenic acid intake reduce the risk of coronary heart disease? A review of the evidence. Altern Ther Health Med. 2005; 11(3):24-30.

12. Micallef MA, Garg ML. Anti-inflammatory and cardioprotective effects of n-3 polyunsaturated fatty acids and plant sterols in hyperlipidemic individuals. Atherosclerosis. 2009; 204(2):476-82. http://dx. doi.org/10.1016/j.atherosclerosis.2008.09.020

13. Harris WS, Bulchandani D. Why do omega-3 fatty acids lower serum triglycerides? Curr Opin Lipidol. 2006; 17(4):387-93. http://dx.doi.org/ 10.1097/01. mol.0000236363.63840.16

14. McLennan PL. Myocardial membrane fatty acids and the antiarrhythmic actions of dietary fish oil in animal models. Lipids. 2001; 36(Suppl.):S111-4. http://dx.doi.org/10.1007/s11745-001-0692-x

15. Leaf A, Kang JX, Xiao YF, Billman GE. Clinical prevention of sudden cardiac death by $n-3$ polyunsaturated fatty acids and mechanism of prevention of arrhythmias by $n-3$ fish oils. Circulation. 2003; 107(21):2646-52. http:// dx.doi.org/10.1161/01.CIR.0000069566.7830 5.33

16. Mozaffarian D, Rimm EB. Fish intake, contaminants, and human health: Evaluating the risks and the benefits. Jama. 2006; 296(15):1885-99. http://dx. doi.org/10.1001/jama.296.15.1885

17. Din JN, Archer RM, Harding SA, Sarma J, Lyall K, Flapan $A D$, et al. Effect of $\omega$-3 fatty acid supplementation on endothelial function, endogenous fibrinolysis and platelet activation in male cigarette smokers. Heart. 2013; 99(3):168-74. http://dx.doi.org/10.1136/heartjnl-2012-302924
18. Nimura N, Kinoshita T. Fluorescent labeling of fatty Acids With 9-Anthryldiazomethane (ADAM) for high performance liquid chromatography. Analytical Letters. 1980; 13(3):191-202. http://dx. doi.org/10.1080/00032718008082552

19. U.S. Department of Health and Human Services. Reducing the health consequences of smoking: 25 years of progress. A Report of the Surgeon General. Rockwille (MD): U.S. Department of Health and Human Services; 1989.

20. Ezzati M, Lopez AD, Rodgers A, Murray CJL. Comparative quantification of health risks: Global and regional burden of disease attributable to selected major risk factors. Geneva: WHO; 2004. http://dx.doi.org/10.1371/journal.pmed.0010027

21. Ebrahimi M, Ghayour-Mobarhan M, Rezaiean S, Hoseini M, Parizade SM, Farhoudi F, et al. Omega3 fatty acid supplements improve the cardiovascular risk profile of subjects with metabolic syndrome, including markers of inflammation and auto-immunity. Acta Cardiol. 2009; 64(3):321-7. http:// dx.doi.org/10.2143/AC.64.3.2038016

22. Jacobson TA, Glickstein SB, Rowe JD, Soni PN. Effects of eicosapentaenoic acid and docosahexaenoic acid on low-density lipoprotein cholesterol and other lipids: A review. J Clin Lipidol. 2012; 6(1):5-18. http://dx.doi.org/10.1016/j.jacl. 2011.10.018

23. Mora S, Otvos JD, Rifai N, Rosenson RS, Buring JE, Ridker PM. Lipoprotein particle profiles by nuclear magnetic resonance compared with standard lipids and apolipoproteins in predicting incident cardiovascular disease in women. Circulation. 2009; 119(7):931-9. http://dx.doi.org/10.1161/CIRCULA TIONAHA. 108.816181

24. de Roos B, Geelen A, Ross K, Rucklidge G, Reid M, Duncan $G$, et al. Identification of potential serum biomarkers of inflammation and lipid modulation that are altered by fish oil supplementation in healthy volunteers. Proteomics. 2008; 8(10):1965-74. http://dx.doi.org/10.1002/pmic.200700457

25. Neff LM, Culiner J, Cunningham-Rundles S, Seidman C, Meehan D, Maturi J, et al. Algal docosahexaenoic acid affects plasma lipoprotein particle size distribution in overweight and obese adults. J Nutr. 2011; 141(2):207-13. http://dx.doi.org/10.3945/jn. 110.130021

26. Kelley DS, Siegel D, Vemuri M, Mackey BE. Docosahexaenoic acid supplementation improves fasting and postprandial lipid profiles in hypertriglyceridemic men. Am J Clin Nutr. 2007; 86(2):324-33. http://dx.doi.org/10.3945/jn. 108. 100354

27. Erkkilä AT, Schwab US, Lehto S, de Mello VD, Kangas AJ, Soininen $P$, et al. Effect of fatty and lean fish intake on lipoprotein subclasses in subjects 
with coronary heart disease: A controlled trial. J Clin Lipidol. 2014; 8(1):126-33. http://dx.doi.org/ 10.1016/j.jacl.2013.09.007

28. Kontush A, Lindahl M, Lhomme M, Calabresi L, Chapman MJ, Davidson WS. Structure of HDL: Particle subclasses and molecular components. Handb Exp Pharmacol. 2015; 224:3-51. http://dx. doi.org/10.1007/978-3-319-09665-0_1

29. Ineu ML, Manenti E, da Costa JLV, Moriguchi E. Manejo da HDL: avanços recentes e perspectivas além da redução de LDL. Arq Bras Cardiol. 2006;
87(6):788-94. http://dx.doi.org/10.1590/S0066-78 2X2006001900017

30. Mostad IL, Bjerve KS, Lydersen S, Grill V. Effects of marine n-3 fatty acid supplementation on lipoprotein subclasses measured by nuclear magnetic resonance in subjects with type II diabetes. Eur J Clin Nutr. 2008; 62(3):419-29. http:// dx. doi.org/10.1038/sj.ejcn. 1602703

Recebido: novembro 6, 2015 Versão final: abril 7, 2016 Aprovado: abril 27, 2016 